\title{
A Comparative Proteome Analysis of Escherichia coli $\Delta$ relA Mutant Cells
}

\author{
Sónia Carneiro ${ }^{1}$, Silas Villas-Bôas ${ }^{2}$, Eugénio C. Ferreira ${ }^{1}$ and Isabel Rocha ${ }^{1 *}$ \\ ${ }^{1}$ CEB - Centre of Biological Engineering, University of Minho, Braga, Portugal, ${ }^{2}$ Centre for Microbial Innovation, School of \\ Biological Sciences, The University of Auckland, Auckland, New Zealand
}

OPEN ACCESS

Edited by:

Xiao-Jun Ji,

Nanjing University of

Technology, China

Reviewed by:

Zhi-Gang Jeff Qian,

Shanghai Jiao Tong University, China

Chang Dou,

University of Washington, USA

${ }^{*}$ Correspondence:

Isabel Rocha

irocha@deb.uminho.pt

Specialty section:

This article was submitted to Process and Industrial Biotechnology, a section of the journal Frontiers in Bioengineering and Biotechnology

Received: 18 May 2016 Accepted: 26 September 2016 Published: 27 October 2016

Citation:

Carneiro S, Villas-Bôas S, Ferreira EC and Rocha I (2016) A Comparative Proteome Analysis of Escherichia coli $\Delta$ relA Mutant Cells.

Front. Bioeng. Biotechnol. 4:78. doi: 10.3389/fbioe.2016.00078
The bacterial RelA-dependent stringent response exerts a strong influence over various processes. In this work, the impact of the relA gene mutation in Escherichia coli cells was evaluated by a quantitative proteomics analysis, employing stable-isotope labeling and high-resolution mass spectrometry. Chemostat cultures of E. coli W3110 and $\Delta$ relA mutant strains were performed at two dilution rates $\left(0.1\right.$ and $\left.0.2 \mathrm{~h}^{-1}\right)$ to assess the influence of the re/A gene mutation in steady-state protein levels. A total of 121 proteins showed significant alterations in their abundance when comparing the proteome of mutant to wild-type cells. The relA gene mutation induced changes on key cellular processes, including the amino acids and nucleotide biosynthesis, the lipid metabolism, transport activities, transcription and translation processes, and responses to stress. Furthermore, some of those changes were more pronounced under specific growth conditions, as the most significant differences in protein ratios were observed at one of the dilution rates. An effect of the relA gene mutation in the acetate overflow was also observed, which confers interesting characteristics to this mutant strain that could be useful in the production of recombinant proteins. Overall, these results provide a valuable insight into the $E$. coli stringent response under defined steady-state conditions, suggesting that this stress response might influence multiple metabolic processes like the acetate overflow or the catabolite repression.

Keywords: quantitative proteomics, iTRAQ analysis, RelA, stringent response, proteome profiling

\section{INTRODUCTION}

The ribosome-bound RelA enzyme is the main bacterial synthetase for guanosine tetraphosphate (ppGpp), a molecule that is at the core of the stringent response. This response is triggered by the deprivation of intracellular amino acids and is characterized by rapid alterations in transcriptional activities, whereby genes required for amino acid biosynthesis are upregulated and genes associated with cell growth are repressed. The coordination of these transcriptional shifts is now understood in some detail and many of the alterations in cellular processes have been elucidated (Chang et al., 2002; Durfee et al., 2008; Traxler et al., 2008). For example, the synthesis of ribosomal proteins was shown to be decreased during the stringent response, involving a combination of the transcriptional and translational control at the level of mRNA stability, i.e., certain ribosomal proteins are self-regulated by decreasing the translation rate of their transcripts (Chang et al., 2002).

Although the effects of relA gene mutations on the global gene expression have been tested, most studies have been focused on the response of Escherichia coli cells under growth transitions caused by glucose-lactose diauxie (Chang et al., 2002; Traxler et al., 2008), $\mathrm{H}_{2} \mathrm{O}_{2}$ treatment (Chang et al., 2002), or the addition of chemical analogs that mimics amino acid starvation, such as serine hydroxymate 
(Durfee et al., 2008). These transient shifts give only temporary and usually growth-dependent gene expression changes in response to environmental alterations, which bias the characterization of physiological states. Continuous cultures remove these transient growth effects and provide a useful tool to address fundamental questions related to elementary microbial processes, such as growth and stress responses.

Previous work on E. coli chemostat cultures (Carneiro et al., 2012) has shown that the RelA activity is growth rate-dependent and differently affects the metabolism of slow- and fast-growing cultures. As such, it is relevant to understand the influence of the RelA activity in the coordination of metabolic processes under different growth conditions. It is anticipated that the relA gene mutation will affect central metabolic processes, as it was previously reported (Carneiro et al., 2011), but the mechanisms by which these processes are regulated and which other entities/processes are involved is still vague. A recent study (Kanjee et al., 2012) has identified direct targeted proteins by the ppGpp alarmone, which explains to a certain extent the global physiological changes in the cellular metabolism.

In this study, a quantitative proteomic analysis, employing stable-isotope labeling and high-resolution mass spectrometry, was used to characterize E. coli cultures under steady-state conditions. This method has gained interest lately due to its high level of proteome coverage, accuracy in protein quantification, and highthroughput (Chong et al., 2006; Wiese et al., 2007; Schwacke et al., 2009; Karp et al., 2010; Kristjansdottir and Kron, 2010; Savitski et al., 2010). The differential labeling of proteins or proteolytic peptides by stable isotopes, followed by relative and absolute quantification by MS analysis is the basis for the determination of concentration ratios of proteins expressed in cells with different phenotypes, e.g., wild-type versus mutant. In our study, 8-plex isobaric tags for absolute and relative quantitation (iTRAQ) were used for the simultaneous relative quantification of peptides from up to eight different samples. Samples from chemostat cultivations with $E$. coli strains W3110 and $\Delta$ relA mutants at two dilution rates were tested. The dilution rates of 0.1 and $0.2 \mathrm{~h}^{-1}$ were chosen in this study because they present distinct metabolic phenotypes as previously verified in our laboratory (Carneiro et al., 2011, 2012). In particular, at a dilution rate of $0.2 \mathrm{~h}^{-1}$, wild-type $E$. coli cells tend to accumulate acetate, which is characteristic of the overflow metabolism. This metabolic behavior is very relevant in recombinant bioprocesses, where the accumulation of acetate hampers protein productivity, decreasing the cost-effectiveness of these processes. Recently, it was detected that strains devoid of RelA activity can decrease the accumulation of acetate (Carneiro et al., 2012), which indicates that recombinant bioprocesses can benefit from the use of $\Delta r e l A$ mutants strains. In order to have a comprehensive overview of the RelA activity in E. coli cells, we analyzed the set of up and downregulated proteins under different steady-state conditions and explored the influence of the $\Delta r e l \mathrm{~A}$ mutation in various cellular processes.

\section{MATERIALS AND METHODS}

\section{Bacterial Strains and Growth Conditions}

Escherichia coli K-12 W3110 (F-, LAM-, IN [rrnD-rrnE]1, rph-1) and the isogenic mutant $\Delta r e l \mathrm{~A}$, obtained from M. Cashel (Xiao et al., 1991), were cultivated in continuous bioreactors at $37^{\circ} \mathrm{C}$, $\mathrm{pH} 7$, and dissolved oxygen above $30 \%$ under fixed dilution rates: 0.1 and $0.2 \mathrm{~h}^{-1}$. Steady-state conditions were verified by constant optical density and glucose measurements. The $\mathrm{pH}$ of the culture was maintained at 7.0 by adding $2.0 \mathrm{M} \mathrm{NaOH}$ or $2.0 \mathrm{M} \mathrm{HCl}$. Dissolved oxygen was maintained above $30 \%$ saturation through a cascade mode controlling the airflow and agitation speed. Chemostat cultivations were operated in a $3 \mathrm{~L}$ fermentor (BioFlo 3000, New Brunswick Scientific, USA) with a working volume of $1.5 \mathrm{~L}$. The working volume was kept constant by withdrawing the culture broth through level control. Cultures were fed with a defined minimal medium consisting of $5 \mathrm{~g} \mathrm{~kg}^{-1}$ of glucose, $6 \mathrm{~g} \mathrm{~kg}^{-1}$ of $\mathrm{Na}_{2} \mathrm{HPO}_{4}, 3 \mathrm{~g} \mathrm{~kg}^{-1}$ of $\mathrm{KH}_{2} \mathrm{PO}_{4}, 0.5 \mathrm{~g} \mathrm{~kg}^{-1}$ of $\mathrm{NaCl}, 1 \mathrm{~g} \mathrm{~kg}^{-1}$ of $\mathrm{NH}_{4} \mathrm{Cl}, 0.015 \mathrm{~g} \mathrm{~kg}^{-1}$ of $\mathrm{CaCl}_{2}, 0.12 \mathrm{~g} \mathrm{~kg}^{-1}$ of $\mathrm{MgSO}_{4} \cdot 7 \mathrm{H}_{2} \mathrm{O}, 0.34 \mathrm{~g}$ $\mathrm{kg}^{-1}$ of thiamine, $2 \mathrm{~mL} \mathrm{~kg}^{-1}$ of trace-element solution [described elsewhere (Rocha and Ferreira, 2002)], and $2 \mathrm{~mL} \mathrm{~kg}^{-1}$ of vitamins solution [described elsewhere (Rocha and Ferreira, 2002)]. The medium was continuously fed, at least for five residence times, at a given dilution rate. The minimal medium was further supplemented with $20 \mathrm{mg} \mathrm{kg}^{-1}$ of $\mathrm{L}$-isoleucine to grow the W3110 strain, while the same medium with further addition of $20 \mathrm{mg} \mathrm{kg}^{-1}$ of L-valine and $25 \mathrm{mg} \mathrm{kg}^{-1}$ of kanamycin was used to grow the $\Delta$ relA mutant strain.

\section{Analytical Techniques}

Biomass was determined by measuring culture turbidity $\left(\mathrm{OD}_{600 \mathrm{~nm}}\right)$ and cell dry weight $(\mathrm{CDW})$. In order to determine CDW, $10 \mathrm{~mL}$ of broth were filtered by $0.2 \mu \mathrm{m}$ filters and the retentate was dried in the microwave to a constant weight. For glucose and acetate analysis, culture broth was centrifuged at $7500 \times g$ for $15 \mathrm{~min}$ to remove the cell debris and the supernatant was collected. The glucose concentration in the culture broth was determined by the dinitrosalicylic acid (DNS) colorimetric method (Miller, 1959). The concentrations of acetic acid in the culture broth were determined with an enzymatic test kit (Acetic acid Test kit, R-Biopharm AG, Germany).

\section{Protein Extraction, Digestion, and ITRAQ Labeling}

Aliquots of biomass from cultures ( $1 \mathrm{~mL}$ ) (in duplicate) were centrifuged and the pellets were suspended in $7 \mathrm{M}$ urea, $2 \mathrm{M}$ thiourea, $10 \mathrm{mM}$ DTT, and 0.1\% SurfactAmps X-100. They were then sonicated on ice during 30-s bursts using a Soniprep 150 probe sonicator (MSE, London, UK). Samples were again centrifuged at $16,000 \times g$, and the supernatants were harvested. Protein content was assayed by the EZQ method (Invitrogen) using 20 -fold dilutions of the supernatants. Aliquots containing $50 \mu \mathrm{g}$ of total protein underwent reduction (incubation at $40^{\circ} \mathrm{C}$ for $1 \mathrm{~h}$ ) and alkylation (incubation with $50 \mathrm{mM}$ iodoacetamide at $\mathrm{pH} 8.0$ in the dark for $1 \mathrm{~h}$ ) followed by quenching with further DTT. Samples were diluted 10 -fold in $50 \mathrm{mM}$ ammonium bicarbonate and digested by incubation with $2 \mu \mathrm{g}$ of trypsin (Promega, Madison, WI, USA) at $37^{\circ} \mathrm{C}$ overnight. The resulting peptides were desalted on $10 \mathrm{mg}$ Oasis SPE cartridges (Waters Corporation, MA, USA) and completely dried using a speed vacuum (Speedvac) concentrator (Thermo Savant, Holbrook, NY, USA). Dried protein digests were re-constituted with $30 \mu \mathrm{L}$ of Dissolution Buffer from the iTRAQ Reagent Multi-Plex Kit (Applied Biosystems, Foster 
City, CA, USA) and labeled with 8-plex iTRAQ reagents according to the manufacturer's instructions. Labeled material from eight different samples was then combined into one sample mixture, acidified and desalted again as above, concentrated in a Speedvac to approximately $50 \mu \mathrm{L}$, and finally diluted to $250 \mu \mathrm{L}$ with $0.1 \%$ formic acid in $10 \%$ acetonitrile.

\section{HPLC-SCX Fractionation and Mass Spectrometry Analysis}

The labeled peptides were separated from the complex mixture according to cationic charge using the strong cation-exchange (SCX) fractionation. The SCX fractionation was performed on a BioSCX II $0.3 \mathrm{~mm} \times 35 \mathrm{~mm}$ column (Agilent Technologies, Santa Clara, CA, USA) using 10 salt-steps: 10, 20, 40, 60, 80, 100, $125,150,200$, and $500 \mathrm{mM} \mathrm{KCl}$. Peptide fractions were captured on a $0.3 \mathrm{~mm} \times 5 \mathrm{~mm}$ PepMap cartridge (LC Packings, Dionex Corporation, Sunnyvale, CA, USA) before being separated on a $0.3 \mathrm{~mm} \times 100 \mathrm{~mm}$ Zorbax 300SB-C18 column (Agilent) by highperformance liquid chromatography (HPLC). The HPLC gradient between buffer A ( $0.1 \%$ formic acid in water) and buffer B $(0.1 \%$ formic acid in acetonitrile) was formed at $6 \mu \mathrm{L} / \mathrm{min}$ as follows: $10 \% \mathrm{~B}$ for the first $3 \mathrm{~min}$, increasing to $35 \% \mathrm{~B}$ until $85 \mathrm{~min}$, increasing to $95 \%$ B until $90 \mathrm{~min}$, held at $95 \%$ until $93 \mathrm{~min}$, back to $10 \% \mathrm{~B}$ at $96 \mathrm{~min}$, and held there until $100 \mathrm{~min}$. The LC effluent was directed into the IonSpray source of a QSTAR XL hybrid Quadrupole-Time-of-Flight mass spectrometer (Applied Biosystems) scanning from 300 to $1600 \mathrm{~m} / z$. The top three most abundant multiply charged peptides were selected for MS/MS analysis $(55-1600 \mathrm{~m} / \mathrm{z})$. The mass spectrometer and the HPLC system were under the control of the Analyst QS software package (Applied Biosystems).

\section{Protein Identification and Quantification}

Peptide identification and quantification were performed using the ProteinPilot software packages (Applied Biosystems). Each MS/MS spectrum was searched against the NCBI (National Center for Biotechnology Information) protein database (http://www. ncbi.nlm.nih.gov/) containing 43657 E. coli $\mathrm{K}-12$ sequences, and protein identification was accepted based on ProteinPilot confidence scores. The search parameters were $95 \%$ confidence for the protein identification threshold, trypsin as digest agent, iodoacetamide as cysteine alkylation, urea denaturation as special factors, and rapid ID as search effort. The detected protein threshold [Unused ProtScore (UPS), i.e., a measure of the protein confidence for a detected protein calculated from the peptide confidence for peptides from spectra that have not already been completely "used" by higher scoring winning proteins] was set to 1.3 to achieve $95 \%$ of confidence and resulted in the identification of 5511 peptides being matched to 536 distinct proteins. The spectra were also searched against the same set of protein sequences in reverse to estimate the false discovery rate (FDR), which resulted in just one protein match with an "unused score" of 1.51, giving a protein identification with a FDR below $0.2 \%$.

Using ProteinPilot, the relative abundance of each peptide in duplicate samples from $\Delta$ relA mutants cells versus wild-type cells grown at $D=0.1 \mathrm{~h}^{-1}$ was determined by dividing signature-ion peak areas at $m / z 114$ and 118 by signature-ion peak areas at $\mathrm{m} / z 113$ and 117 , respectively (corresponding to the labels chosen for each sample, i.e., 114 and 118 correspond to the tags chosen for the two replicates of mutant cells at $D=0.1 \mathrm{~h}^{-1}$ while 113 and 117 correspond to the tags chosen for the wild-type also at $D=0.1 \mathrm{~h}^{-1}$ ). Similarly, the level of peptides in duplicate samples from $\Delta$ relA mutant cells at $D=0.2 \mathrm{~h}^{-1}$ and wild-type grown at $D=0.2 \mathrm{~h}^{-1}$ was calculated by dividing signature-ion peak areas at $m / z 116$ and 121 by signature-ion peak areas at $m / z 115$ and 119 , respectively.

The ProGroup Algorithm (within ProteinPilot) was used to compile the results from the database search into protein groups and to report protein-based ratios of relative abundance for each condition. ProteinPilot calculates average iTRAQ ratios and estimates the $p$-value and error factor (EF) for each protein hit. Only proteins identified with a minimum of two peptides and EF values lower than 2 were considered for protein identification and relative quantification. The EF defines the quantitative $95 \%$ confidence interval (CI) of a ratio as follows:

$$
\mathrm{EF}=10^{95 \% \mathrm{CI}}
$$

where

$$
\text { 95\% CI }=\text { Protein ratio }(R) \times \mathrm{EF}-\frac{\text { Protein ratio }(R)}{\mathrm{EF}}
$$

All protein ratios were converted to log space, and the average protein expression was estimated using the following equation adapted from the ProQuant software tutorial:

$$
R_{w}=\frac{\sum\left(w_{i} \times x_{i}\right)}{\sum w_{i}}
$$

where $w_{i}=1 /($ EF of the protein), the weight for the $i$ th peptide and $x_{i}=\log _{2}$ (protein ratio), the ratio for the $i$ th peptide in log scale. The weighted $\mathrm{SD}_{w}$ was estimated using the following equations also adapted from the ProQuant software tutorial:

$$
\mathrm{SD}_{w}=\frac{\mathrm{SD}}{b^{0.5}}
$$

where SD is the unweighted SD and $b=\frac{\left(\sum_{i=1}^{N} w_{i}\right)^{2}}{\sum_{i=1}^{N} w_{i}^{2}}$. For the selection of differentially expressed proteins, we considered only proteins with average expression weighted log ratios larger than 0.26 and lower than -0.32 , and with an $\mathrm{SD}_{\mathrm{w}}$ inferior to 1.5 times the corresponding ratio.

\section{Functional Enrichment and Construction of a Regulatory Subnetwork}

We have also examined the enrichment of gene ontology (GO) biological processes in the differentially expressed proteins. The GOToolBox (Martin et al., 2004) was used to retrieve overrepresented GO terms related to biological processes in up and downregulated proteins. The following settings were used: annotation set - EcoCyc and EcoliWiki collaborative annotation for E. coli $\mathrm{K}-12$; ontology - biological process; mode - all terms; reference genome; evidence - all evidence codes. 
Next, a base-network consisting in gene regulatory interactions was constructed based on transcriptional regulatory information obtained from RegulonDB (Salgado et al., 2012). This basenetwork was then used to construct subnetworks by filtering all interactions where at least one of the gene-coding proteins with significant alterations in their abundance between cultures (i.e., mutant and wild-type) was participating. Such an approach yielded two subnetworks corresponding to each experimental condition, i.e., dilution rates of 0.1 and $0.2 \mathrm{~h}^{-1}$, consisting in a total of 95 and 306 nodes, and 160 and 359 regulatory interactions, respectively.

\section{RESULTS}

Physiological parameters were measured in both culturing conditions (dilution rates of 0.1 and $0.2 \mathrm{~h}^{-1}$ ), for strains W3110 and $\Delta$ relA showing that biomass yields were lower in the wild-type culture for the $0.2 \mathrm{~h}^{-1}$ dilution rate, although the differences are not significant, given the CIs. Acetate accumulation was considerably higher in the wild-type culture compared to the $\Delta$ relA culture at a dilution rate of $0.2 \mathrm{~h}^{-1}$ (Table 1 ).

Using ProteinPilot, 5511 peptides were identified and matched with the NCBI protein database from which 536 protein candidates were found. After analysis, 70 proteins show a significant increase in abundance in mutant cells when compared to wild-type cells, while 46 proteins showed a significant decrease in abundance, as listed in Table S1 in Supplementary Material. This indicates that $\sim 22 \%$ of the identified proteins changed significantly their abundance and, hence, may play a role in RelA-dependent response.

The functional enrichment of the differentially expressed proteome was then examined using GO terms of biological processes. GO terms, such as translation (GO:0006412), protein metabolic process (GO:0019538), reproductive process (GO:0022414), protein folding (GO:0006457), homoserine biosynthetic process (GO:0009090), cellular amino acid biosynthetic process (GO:0008652), cellular amine metabolic process

TABLE 1 | Growth parameters of the wild-type and $\triangle$ relA mutant E. coli strains in aerobic glucose-limited continuous culture.

\begin{tabular}{lcccc}
\hline & \multicolumn{2}{c}{ Wild type } & \multicolumn{2}{c}{$\Delta$ relA mutant } \\
\hline $\begin{array}{l}\text { Dilution } \\
\text { rate }\left(\mathrm{h}^{-1}\right)\end{array}$ & 0.10 & 0.20 & 0.10 & 0.20 \\
$\begin{array}{l}\text { Biomass yield } \\
\left(\mathrm{g} \mathrm{g}^{-1}\right)\end{array}$ & $0.44 \pm 0.15$ & $0.55 \pm 0.10$ & $0.46 \pm 0.06$ & $0.67 \pm 0.30$ \\
$\begin{array}{l}\text { Biomass } \\
\left(\mathrm{g} \mathrm{L}^{-1}\right)\end{array}$ & $2.2 \pm 0.3$ & $2.7 \pm 0.4$ & $2.3 \pm 0.3$ & $3.3 \pm 0.5$ \\
Glucose $\left(\mathrm{gL}^{-1}\right)$ & $0.029 \pm 0.009$ & $0.040 \pm 0.003$ & $(1)$ & $0.023 \pm 0.010$ \\
$\begin{array}{l}q_{\text {Glucose }} \\
\left(\mathrm{g} \mathrm{g}^{-1} \mathrm{~h}^{-1}\right)\end{array}$ & $0.23 \pm 0.08$ & $0.36 \pm 0.06$ & $0.22 \pm 0.03$ & $0.30 \pm 0.13$ \\
Acetate $\left(\mathrm{g} \mathrm{L}^{-1}\right)$ & $(1)$ & 0.34 & $(1)$ & 0.02 \\
$q_{\text {Acetate }}\left(10^{-3}\right)$ & - & $25.0 \pm 3.8$ & - & $1.1 \pm 0.2$ \\
$\left(\mathrm{~g} \mathrm{~g}^{-1} \mathrm{~h}^{-1}\right)$ & & & & \\
\hline
\end{tabular}

(1) Undeterminable traces.
(GO:0009308), detection of biotic stimulus (GO:0009595), and lysine biosynthetic process (GO:0009085), were highlighted in the analysis. Figure 1 shows the most representative GO terms associated with biological processes for proteins with significant alterations in mutant cells.

Next, we have constructed gene regulatory subnetworks to explore the connectivity of proteins with significant alterations in their abundance induced by the $\Delta$ relA gene mutation under different steady-state conditions (see Figure 2). These subnetworks allowed us to identify cellular processes that were affected by the gene mutation and whose regulation is closely related at specific growth conditions. For instance, proteins within the same regulatory module are most likely to be involved in the RelA-dependent stringent response.

In total, we have found $\sim 121$ differentially expressed proteins in each experimental condition, which involved 160 regulatory interactions for the dilution rate of $0.1 \mathrm{~h}^{-1}$ (Figure 2A) and almost 400 regulatory interactions for the dilution rate of $0.2 \mathrm{~h}^{-1}$ (Figure 2B). The increase in regulatory interactions was essentially due to the significant upregulation of regulatory proteins, such as $\mathrm{IhfB}$ and $\mathrm{RcsB}$ in the mutant culture at a higher dilution rate, which can affect a larger number of other proteins. In turn, at the dilution rate of $0.1 \mathrm{~h}^{-1}$ only one transcriptional regulator was found to be differentially expressed (i.e., HupB) and it regulates up to 10 genes, which resulted in a less structurally complex network. To further explore the proteome changes induced by the $\Delta$ relA gene mutation, we have classified proteins into three groups (Figure 3): (I) proteins that were equally up or downregulated in cultures at both dilution rate conditions $\left(0.1\right.$ and $\left.0.2 \mathrm{~h}^{-1}\right)$; (II) proteins that were differently expressed in only one of the conditions, i.e., either at a dilution rate of 0.1 or $0.2 \mathrm{~h}^{-1}$; and (III) proteins for which the expression ratios were opposite between dilution rate conditions.

\section{Group I}

The first group consists of proteins that were consistently induced or repressed in both dilution rates and, thus, alterations in their protein levels are dependent on the gene mutation rather than on growth conditions. A total of 29 proteins were identified to respond seemingly in both growth conditions and approximately two-thirds presented upregulated levels in mutant cells. Proteins involved in the detection of stimulus (e.g., GadB, GadC, and Slp), response to stress (e.g., ClpB, Dps, HtpG, and YfiD), protein folding (e.g., CbpA, HdeB, DnaK, GroL, and GroS), and oxidative reduction activities (e.g., GrxB, AdhE and CydB) were upregulated in the mutant culture.

\section{Group II}

Within the second group, a total of 41 proteins were exclusively identified in cultures run at a dilution rate of $0.1 \mathrm{~h}^{-1}$, among which more than half (23) were downregulated and 18 were upregulated in mutant cells. At these conditions, proteins associated with transport activities (e.g., OmpF, MalE, MglB, and GatB) were majorly downregulated in mutant cells, whereas proteins related to other metabolic processes, such as lipid biosynthesis (e.g., LpcA, FabA, FabB, and FabD) or nucleotide biosynthesis (e.g., CarA, PurB, and Upp), were upregulated. On the other hand, 46 proteins 


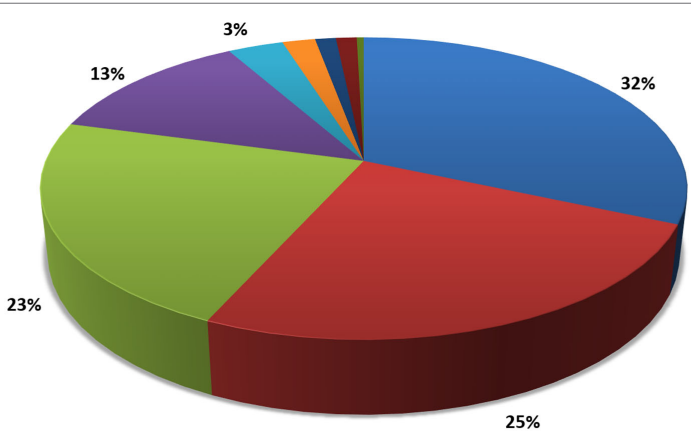

$\begin{array}{lll}\text { Protein metabolic process } & \text { Mellular amine metabolic process } & \square \text { Cellular amino acid metabolic process } \\ \text { Translation } & \text { Protein folding } & \text { Reproductive process } \\ \text { Lysine biosynthetic process } & \text { Detection of biotic stimulus } & \text { Momoserine biosynthetic process }\end{array}$

\begin{tabular}{ll}
\hline Biological process & List of Proteins \\
\hline Protein metabolic process & GroEL, RpsR, RpsS, RplC, ClpB, IhfB, HtpG, GroE, RplR, CbpA, RplF, RpsJ, FtsH, SerS, RplP, \\
& RpsF, RplY, PepD, AspS, RpmB, DnaK, RplX, RplV, HchA, RpmC, DsbA \\
Cellular amine metabolic process & TraA, DapD, LeuA, IlvC, LysC, IscS, GuaA, SerS, GabT, ThrC, GItB, PyrB, Asd, ThrA, AspS, \\
& GltD, MetK, GadB \\
Cellular amino acid metabolic process & TraA, DapD, LeuA, IlvC, LysC, IscS, GuaA, SerS, ThrC, GltB, PyrB, Asd, ThrA, AspS, GltD, \\
& MetK, GadB \\
Translation & RpsR, RpsS, RplC, IhfB, RplR, RplF, RpsJ, SerS, RplP, RpsF, RplY, AspS, RpmB, RplX, RplV, \\
& RpmC \\
Protein folding & GroEL, HtpG, GroE, CbpA, DnaK, HchA, DsbA \\
Reproductive process & GroEL, OmpF, Tsx, GroE, OmpC, OmpA \\
Lysine biosynthetic process & DapD, LysC, Asd, ThrA \\
Detection of biotic stimulus & OmpF, Tsx, OmpC, OmpA \\
Homoserine biosynthetic process & LysC, Asd, ThrA \\
\hline
\end{tabular}

FIGURE 1 | Distribution of the most relevant biological process terms (GO) among proteins that changed in relative abundance between wild-type and $\triangle$ relA mutant cells

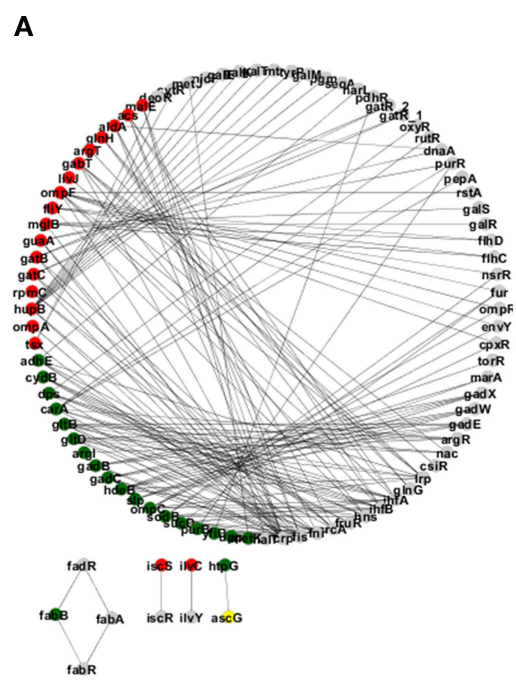

$0.1 \mathrm{~h}^{-1}$

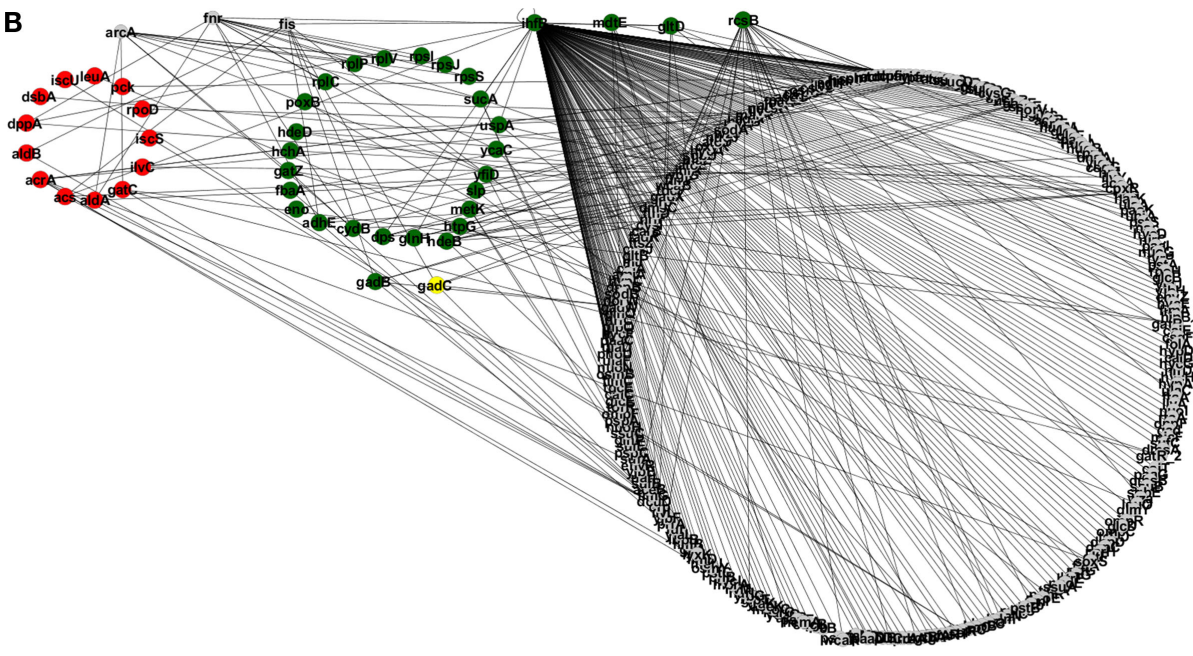

$0.2 \mathrm{~h}^{-1}$

FIGURE 2 | Regulatory subnetworks representing the significant proteome changes between the $E$. coli $\Delta$ relA mutant and wild-type cultures at two steady-state conditions: dilution rates of $\mathbf{0 . 1}(\mathbf{A})$ and $\mathbf{0 . 2} \mathbf{h}^{-\mathbf{1}}$ (B). Upregulated proteins in the mutant culture are represented by green nodes and downregulated proteins are represented by red nodes. Gray nodes represent proteins that were only detected in one of the cultures. 


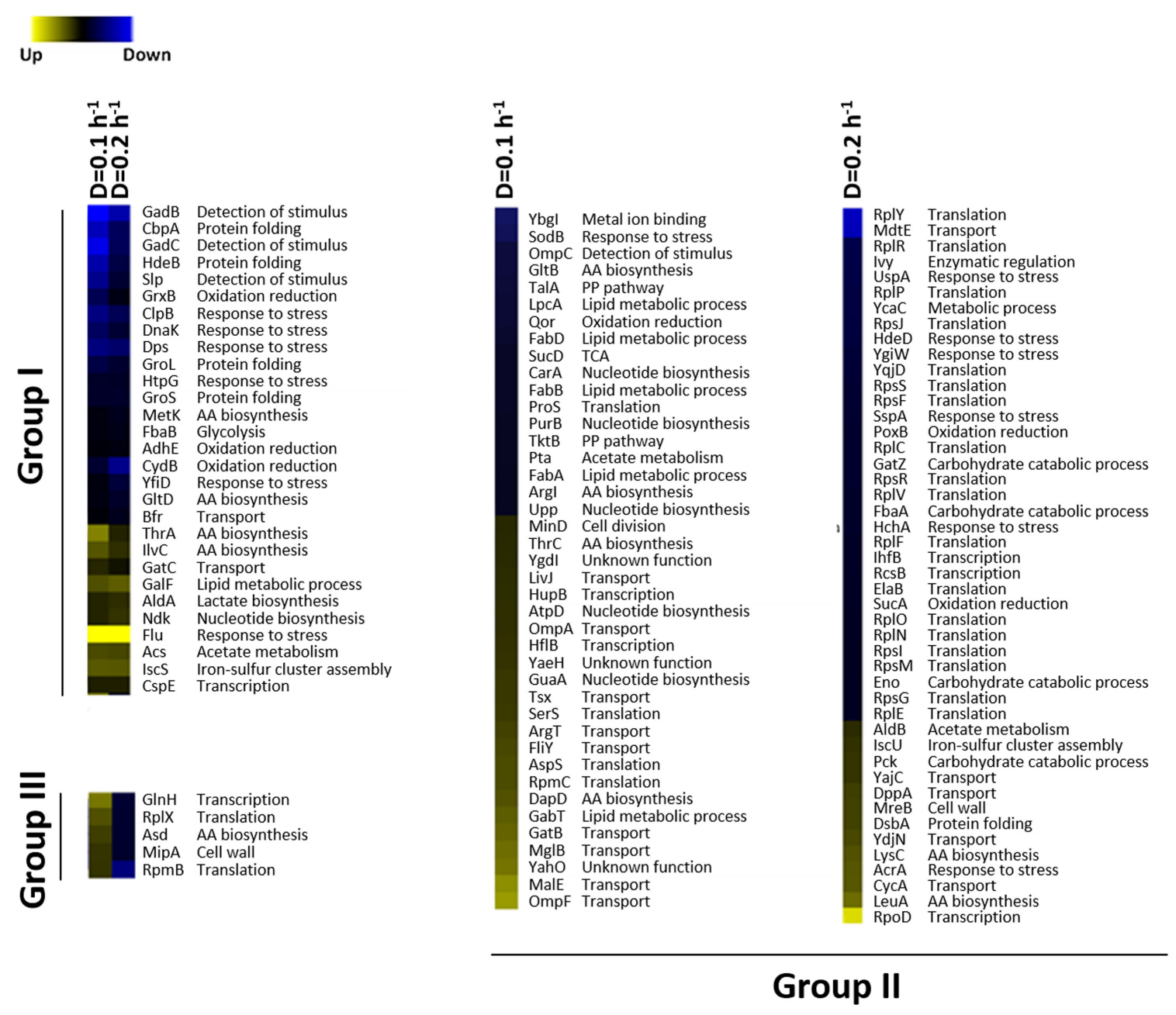

FIGURE 3 | Differential protein expression patterns in the $E$. coli $\Delta$ relA mutant versus wild-type cells at two dilution rates. Proteins included in group I were seemingly expressed in the two dilution rate conditions $\left(0.1\right.$ or $\left.0.2 \mathrm{~h}^{-1}\right)$; group II includes proteins that were detected only in either dilution rates 0.1 or $0.2 \mathrm{~h}^{-1}$; and group III includes proteins for which the steady-state expression levels were opposite between dilution rate conditions. Proteins with significant upregulated expressions in the mutant cultures are colored in blue, while proteins with downregulated levels are in yellow.

were solely identified in cultures run at a dilution rate of $0.2 \mathrm{~h}^{-1}$, comprising 13 proteins with downregulated expression levels in the mutant culture and 33 with upregulated levels. Proteins involved in translation activities (e.g., RplY, RplR, RplP, and RpsJ) and responses to stimulus/stress (e.g., UspA, HdeD, and YgiW) were markedly upregulated in mutant cells, while proteins related with transport (e.g., CycA and YajC) and amino acid biosynthesis (e.g., LeuA and LysC) were downregulated.

\section{Group III}

Finally, in the last group only five proteins were identified, namely $\mathrm{GlnH}$, RplX, Asd, MipA, and RpmB. Interestingly, all proteins showed downregulated expression levels in mutant cells at a dilution rate of $0.1 \mathrm{~h}^{-1}$ and upregulated at $0.2 \mathrm{~h}^{-1}$. These proteins cover cellular functions, such as translation (RplX and RpmB), transport $(\mathrm{Gln} H)$, and metabolic activities, such as the Asd protein that has a role in the homoserine biosynthesis and proteins with scaffolding properties in bacterial murein (MipA).

\section{DISCUSSION}

In this study, we sought to investigate which cellular processes are affected the most by the lack of the RelA-dependent stringent control under steady-state growth conditions. For that purpose, the proteome from continuous $E$. coli cultures were examined using iTRAQ labeling coupled with LC-MS/MS, identifying 121 differentially expressed proteins.

As it can be seen from Figure 1, a large fraction of proteins with functions in translation (GO:0006412), protein (GO:0019538) and cellular amine (GO:0009308) metabolic processes were found to have significant altered protein levels between the wild-type and $\Delta$ relA mutant cultures. These results are in agreement with previous studies (Jain et al., 2006; Durfee et al., 2008; Ferullo and Lovett, 2008; Traxler et al., 2008) that associate the RelAdependent response in $E$. coli cells with various cellular functions, such as translation and amino acid biosynthetic activities.

Although the RelA enzyme is involved in many cellular responses (Wendrich et al., 2002; Srivatsan and Wang, 2008; 
Li et al., 2016), it has been recognized to be central in the bacterial stringent response. Besides this enzyme, other key players have been identified, such as the SpoT bifunctional (Traxler et al., 2008; Wu and Xie, 2009; Hauryliuk et al., 2015). Though the biosynthetic activity of SpoT is less significant than that of RelA, it also contributes to the synthesis of (p)ppGpp, a signaling molecule that triggers most changes in the cell physiology during the stringent response (Xiao et al., 1991). This means that the single relA mutation may not result into a completely (p)ppGppdevoid phenotype, and as such molecular changes in relA mutants cannot be attributed to the lack of a general stringent response, but as a consequence of a deficient or lower accumulation of (p)ppGpp that is essentially RelA-dependent.

In this study, an attempt is made to clarify the RelA-dependent stringent control using a systems-wide approach by monitoring proteomic profiles under different growth conditions. To better characterize the molecular changes directly associated with the cellular stringent response, mutants lacking relA and spoT should be considered in future studies. In addition, the monitoring of (p)ppGpp levels would be relevant to investigate changes in cell physiology directly associated with this response.

The influence of the dilution rate on protein expression ratios allowed to discriminate proteins into three groups (Figure 3). Proteins in group I showed expression levels equally up- and downregulated in both growth conditions, suggesting that alterations in the protein ratios were primarily influenced by the gene mutation. Proteins related to metabolic processes, such as amino acids biosynthesis (ThrA, IlvC,), nucleotide biosynthesis (Ndk), lactate production (AldA), or acetate metabolism (Acs), were persistently downregulated in the mutant cultures, while those related to stress responses (ClpB, Dps, HtpG, and YfiD), detection of stimulus (GadB, GadC, and Slp), or protein folding (CbpA, HdeB, DnaK, GroL, and GroS) were upregulated. It seems that in the absence of the typical RelA-dependent stringent response, alternative stress responses are triggered.

In group II, proteins with expression levels, either significantly up- or downregulated in only one of the growth conditions, were included. Biological processes, such as alternative transport functions, appear to be negatively affected in the mutant culture at $0.1 \mathrm{~h}^{-1}$, revealing that unlike wild-type cells, scavenging for alternative nutrient sources was not elicited by the mutant strain. At $0.2 \mathrm{~h}^{-1}$, some transporters were also downregulated in mutants, but at much less extent, which may be explained by the absence of catabolite repression mechanisms that could trigger the activation of scavenging mechanisms under these conditions. This starvation-like response has been reported in other studies (Brückner and Titgemeyer, 2002; Peterson et al., 2005; Nanchen et al., 2008), associating the overexpression of alternative transporters and the activation of anaplerotic routes with this stress response, which in the absence of catabolite repression mechanisms allows for the use of alternative carbon sources. Apparently, the influence of the RelA activity on the control of catabolite repression mechanisms is significant, since visible alterations in the proteome were observed, in particular the downregulation of proteins associated with alternative catabolic activities in the mutant strain. Although the expression ratios of CRP, the catabolite regulation protein (Nanchen et al.,
2008), were not significantly changed, this regulator acts as a common linker between significantly changed proteins, such as GabT, GatB, GltB, GuaA, HupB, MalE, MglB, OmpA, OmpF, SodB, SucD, and Tsx, suggesting that the regulatory activity of this module is central. Also, as illustrated in Figures 4 and 5, most of the transporters with significant proteins ratios were downregulated in the mutant culture. At these growth conditions, we can assume that the catabolite repression phenomena and, more specifically transport functions, are the most significant alterations in cellular processes derived from the $\Delta$ relA gene mutation.

At a dilution rate of $0.2 \mathrm{~h}^{-1}$, mutant cells showed a significant upregulation of translation processes compared to the wild-type strain. The downregulation of translation functions has been described as a hallmark in the RelA-dependent stringent response (Paul et al., 2004; Durfee et al., 2008; Srivatsan and Wang, 2008). The transcription of ribosomal protein operons is inhibited by the accumulation of ppGpp, which decreases the amount of ribosomes available for protein synthesis (Traxler et al., 2006) and ultimately results in lower biomass formation. Mutants defective in the stringent response, i.e., $\Delta$ relA mutants, do not accumulate high levels of ppGpp and the transcriptional control of these operons is most likely "relaxed." For that reason, $\Delta$ relA mutant cells are often said to have "relaxed" phenotypes (Herman et al., 1994). Although differences in biomass formation between wild-type and $\Delta$ relA mutant cultures were not significant in this study, biomass levels were slightly higher in the $\Delta r e l \mathrm{~A}$ mutant culture at a dilution rate of $0.2 \mathrm{~h}^{-1}$, which could indicate that $\Delta r e l \mathrm{~A}$ mutants are less effective to control translation processes and, thus, growth-related processes are less stringently controlled. On the other hand, acetate production was lower in mutant cultures (having decreased from 0.34 to $0.02 \mathrm{~g} / \mathrm{L}$ in the mutant culture at the dilution rate of $0.2 \mathrm{~h}^{-1}$ ), which may explain biomass levels, since acetate accumulation is known to impair cellular growth. Although it is not entirely understood how acetate overflow and the RelA-stringent control are connected, lower acetate levels in mutants suggest that the absence of the RelAstringent control might reduce metabolic bottlenecks that ultimately lead to the accumulation of by-products, such as acetate. These results are also in agreement with metabolomics profiling analyses performed at the same conditions (Carneiro et al., 2012), where the accumulation of acetate and lactate, another metabolic by-product resulting from the metabolic overflow, was lower in mutant cultures. In this instance, we cannot state that alterations detected in protein ratios related to translation processes are caused by the gene mutation or a consequence of the reduced accumulation of acetate, but clearly the lack of the RelA activity induced profound alterations in many growth-related processes.

Also, the regulatory subnetwork illustrated in Figure 2B shows that the differentially expressed proteome at a dilution rate of $0.2 \mathrm{~h}^{-1}$ is structurally more complex with most proteins being upregulated in the mutant culture, including transcriptional regulators, such as the IhfB that regulates at least 8 of the differentially expressed proteins (Acs, DppA, Dps, GlnH, GltD, IhfB, SucA, and UspA). Clearly, this group comprises a set of proteins with relevant proteomic profiles, suggesting that global changes in the cellular organization of bacterial cells are elicited by the lack of 


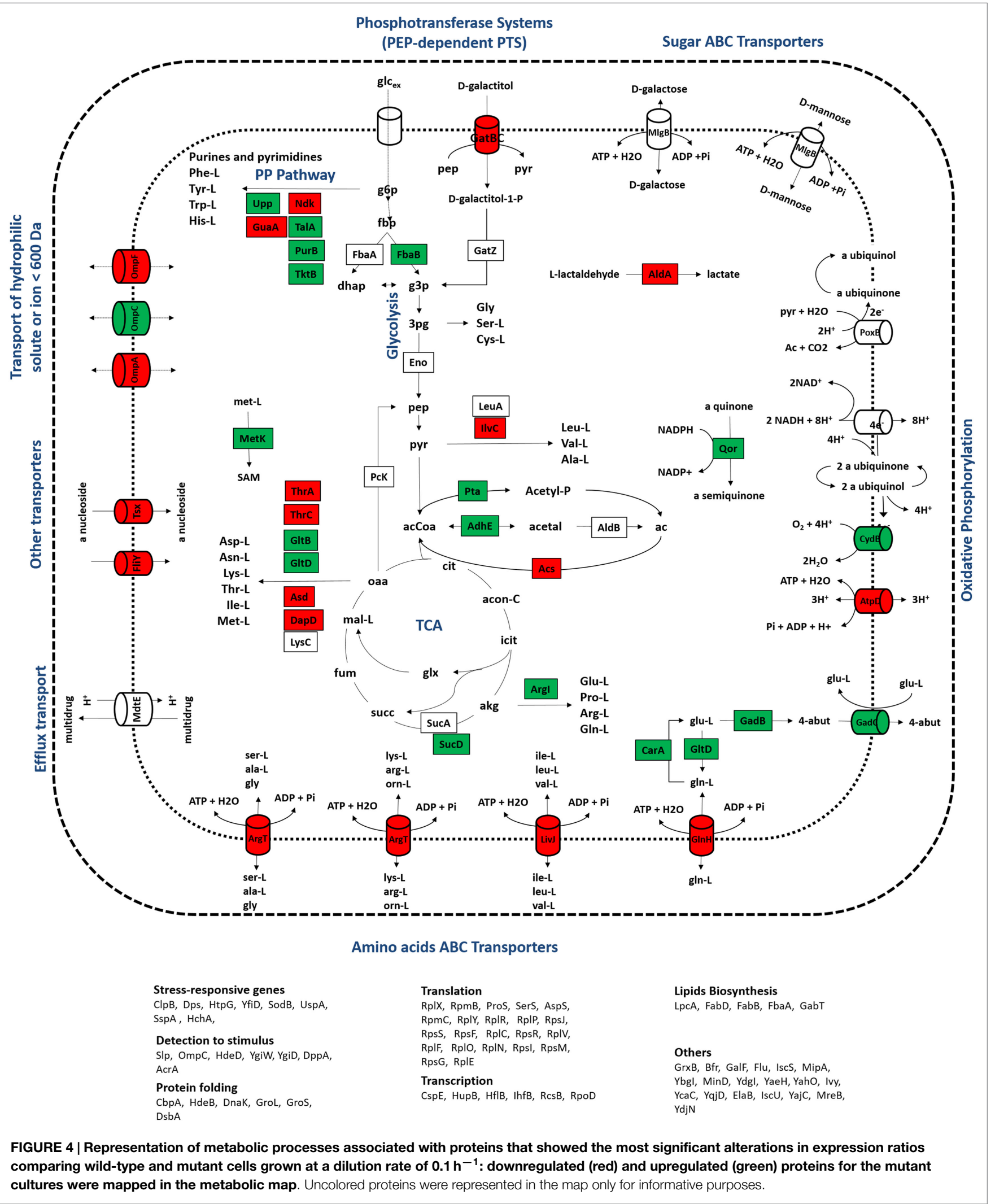




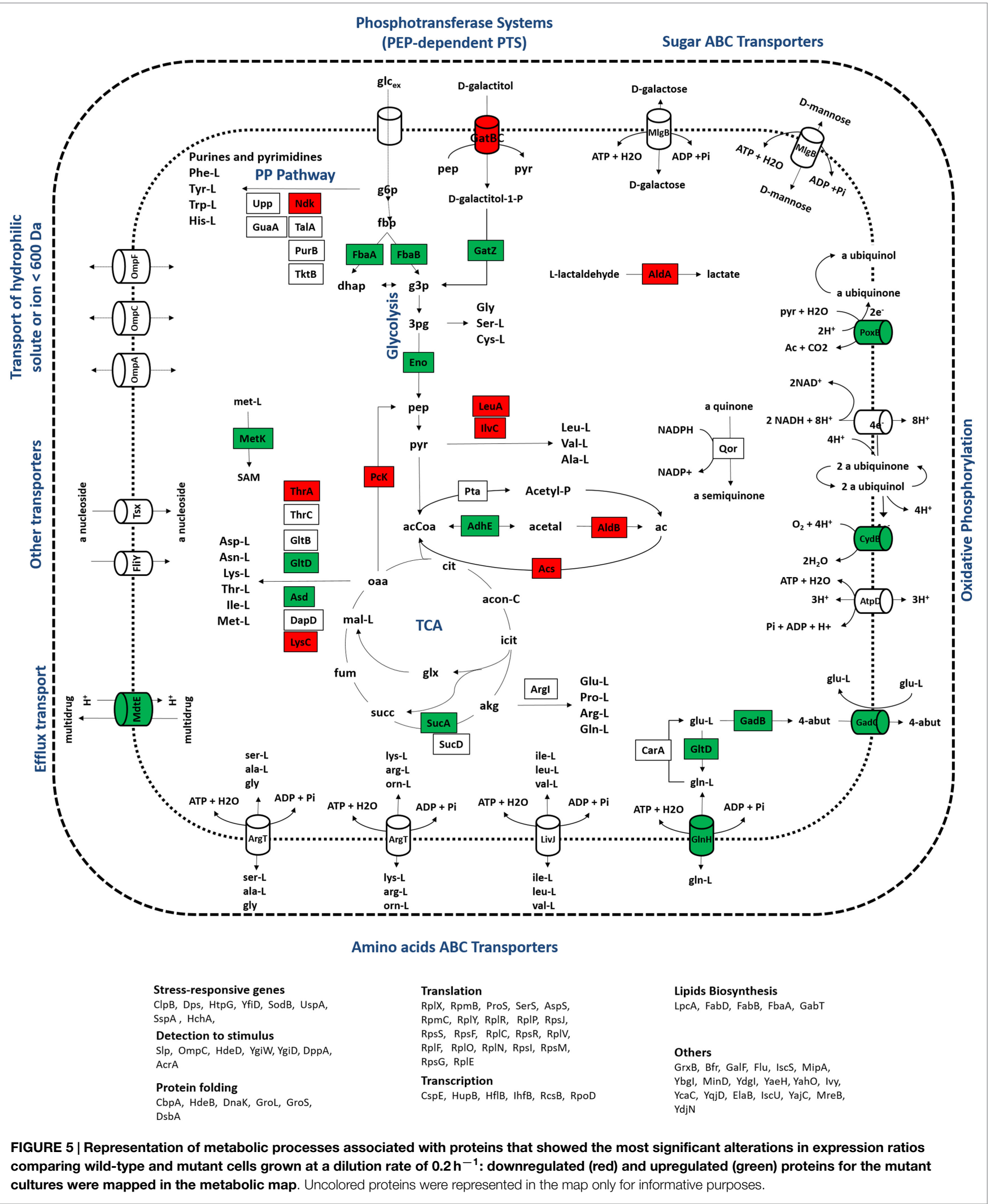


the RelA activity, particularly involving cellular processes, such as translation, metabolism, and stress responses.

In the last group (III), only five proteins were detected to have significant opposite expression ratios: $\mathrm{GlnH}$, RplX, RpmB, Asd, and MipA. All proteins were upregulated in the mutant culture at a dilution rate of $0.2 \mathrm{~h}^{-1}$ and downregulated at $0.1 \mathrm{~h}^{-1}$. This suggests that those proteins have cellular functions that are controlled not only by the RelA-dependent stringent response but also by growth-related processes, meaning that the influence of the RelA can be different at different dilution rates. Here, we cannot ignore the fact that by changing growth conditions different cellular processes may be elicited that intersect the cascade of regulations induced by the RelA-stringent control, which, ultimately, can influence protein expression levels in a different way (Srivatsan and Wang, 2008). For example, RplX and RpmB, two 50S subunits required for ribosome assembly, were upregulated in the mutant culture at a dilution rate of $0.2 \mathrm{~h}^{-1}$, but were downregulated at $0.1 \mathrm{~h}^{-1}$. Presumably, at a dilution rate of $0.2 \mathrm{~h}^{-1}$ the expression of these ribosomal proteins is influenced by the RelA-dependent control, though it is not expected that at this dilution rate the stringent response can be triggered. At a dilution rate of $0.1 \mathrm{~h}^{-1}$ only one translation-related protein (ProS, an aminoacyl-tRNA synthetase) was found to be upregulated in the mutant culture.

Overall, from these results, it can be hypothesized that the RelA activity can influence (directly or indirectly) crucial metabolic processes, such as the catabolite repression control and acetate overflow, that impose large alterations in the metabolic behavior of $E$. coli cells. For instance, mutant cells seem to be unable to respond to starvation-like conditions by inducing nutrient scavenging. Also, the accumulation of acetate was significantly reduced and metabolic activities, such as TCA, lipids, amino acids, and nucleotide biosynthesis, were less impaired, which confers this mutant a suitable phenotype for biosynthetic purposes, e.g., recombinant protein production. Researchers has long been searching for strains with physiological characteristics capable to increase protein productivity, and cells deficient in the stringent response have been indicated as promising recombinant hosts (Schweder et al., 1995).

A final inspection was performed by comparing the proteome profiles with the transcriptional responses of E. coli in other studies related to the RelA-mediated stringent control (Table 2). We sought to address gene products involved in this response by comparing with other experimental studies. Although most of the reported studies are focused on the transcriptional responses of $E$. coli cultures upon alterations in environmental conditions (e.g., diauxie conditions and serine hydroxymate or $\mathrm{H}_{2} \mathrm{O}_{2}$ treatments), the overlap between differentially expressed transcripts found in those studies with gene products in the present proteomics study indicates a close relationship between transcriptional responses to nutrient downshifts or starvation and the RelA-dependent control of protein expression during steady-state conditions. For instance, the downregulation of ald $\mathrm{A}$ in the mutant strain, a gene coding for the aldehyde dehydrogenase enzyme that is involved in the conversion of L-lactaldehyde into lactate, was also reported in Traxler and Chang studies (Traxler et al., 2006) upon glucose-lactose diauxie conditions. Besides gene products with
TABLE 2 | Gene products that are in common with microarray studies.

\begin{tabular}{|c|c|c|}
\hline Reference & Description & Protein \\
\hline $\begin{array}{l}\text { Durfee et al. } \\
\text { (2008) }\end{array}$ & $\begin{array}{l}\text { Time series of gene expression } \\
\text { profiles for two serine } \\
\text { hydroxymate-treated cultures: a } \\
\text { wild-type } E \text {. coli K-12 strain, } \\
\text { and an isogenic } \Delta \text { relA251 } \\
\text { derivative defective in the } \\
\text { stringent response }\end{array}$ & $\begin{array}{l}20 \text { gene products: arg } T, \text { bfr, } \\
c s p E, \text { dnaK, elaB, eno, fbaA, } \\
\text { gatZ, gltA, groL, gros, hupA, } \\
\text { malE, omp } F, \text { pgk, ptsH, rp/Y, } \\
\text { rps } F, \text { sucD, tsx }\end{array}$ \\
\hline $\begin{array}{l}\text { Traxler et al. } \\
(2006)\end{array}$ & $\begin{array}{l}\text { Changes in transcription in } \\
\text { wild-type E. coli and mutants } \\
\text { that lack RelA (ppGpp } \\
\text { synthetase) upon } \\
\text { glucose-lactose diauxie } \\
\text { conditions were determined }\end{array}$ & $\begin{array}{l}12 \text { gene products: acs, aldA, } \\
\text { bfr, dps, elaB, fusA, gatY, } \\
\text { malE, mglB, rplL, rp/R, rplX }\end{array}$ \\
\hline $\begin{array}{l}\text { Chang et al. } \\
(2002)\end{array}$ & $\begin{array}{l}\text { Gene expression profiling of } \\
\text { E. coli growth transitions } \\
\text { caused by glucose-lactose } \\
\text { diauxie, } \mathrm{H}_{2} \mathrm{O}_{2} \text { treatment, and } \\
\text { entry into the stationary phase } \\
\text { to study the stringent control of } \\
\text { genetic systems }\end{array}$ & $\begin{array}{l}26 \text { gene products: ahpC, } \\
\text { aldA, atpA, bfr, cspC, cspE, } \\
\text { dnaK, dps, fusA, gapA, glnH, } \\
\text { gltB, glyA, ilvC, malE, mglB, } \\
\text { ompF, pyrB, rplE, rplL, rplP, } \\
\text { rp/X, rp/Y, rpmB, rpsl, thrC }\end{array}$ \\
\hline
\end{tabular}

metabolic functions, other transcripts were overlapped with the set of significant proteins found in this study. Transcripts associated with transcriptional processes and the translation apparatus were evidenced, most notably the rRNA-coding genes $r p l \mathrm{E}$, $r p l \mathrm{~L}$, $r p l \mathrm{X}$, and $r p l \mathrm{Y}$, and the $c s p \mathrm{E}$ gene, coding for the transcription antiterminator and regulator of RNA stability. It is well-known that genes whose products are primarily involved in the translation apparatus are downregulated during the RelA-stringent control as part of the programed response to growth arrest (Barker et al., 2001; Schneider et al., 2003; Paul et al., 2004; Srivatsan and Wang, 2008). As a result, the expression levels of these genes are strongly reduced in the wild-type cells upon glucose-lactose diauxie compared to the $\Delta$ relA mutant cells. This was also detected in the proteome profiles in this work, where protein levels were higher in the $\Delta$ relA mutant cultures.

Other transcripts involved in protein folding processes were also evidenced: $d n a \mathrm{~K}$ and groS genes coding for molecular chaperones. The expression of these proteins may decrease after inducing stress conditions (Traxler et al., 2006; Durfee et al., 2008), but it seems that cells may not react accordingly to both stress factors. While the serine hydroxymate treatment induced a higher decrease in the expression of chaperones in mutant compared to wild-type cells (Durfee et al., 2008), diauxic conditions exerted a stronger effect on wild-type cells, decreasing even more the expression levels of chaperones (Traxler et al., 2006). The proteome profiles in this study indicate that the upregulation of these gene products was significant in the mutant cells, suggesting that to what relates with protein folding processes the behavior of these cells is closer to changes during diauxic transitions. Overall, the correlation between transcriptional changes in E. coli cells in response to environmental shifts and the proteome profiles detected under steady-state growth conditions of wild-type and $\Delta$ relA mutant cultures suggest that the relA gene mutation has a permanent effect on cellular responses. 


\section{CONCLUSION}

Our scope of interest in this study was not only to explore the differential proteome of the wild-type and $\Delta$ relA mutant $E$. coli cultures but also in particular the effect of this single gene mutation on the steady-state cellular growth. Industrial applications of this mutant strain as a host system have been proposed to improve recombinant protein production and it is fundamental to characterize the mutant phenotype. However, the environmental and growth conditions that stimulate the RelA-dependent stringent response and how a single relA gene mutation affects cellular processes at these conditions are scarcely known.

For this purpose, we investigated the proteome profiles produced by the wild-type and the isogenic $\Delta$ relA mutant cultures at two fixed steady-state conditions (i.e., 0.1 and $0.2 \mathrm{~h}^{-1}$ ). This allowed to investigate the primary effects of the single gene mutation on cellular activities under carefully controlled growth conditions and to evaluate condition-specific regulatory structures that characterize the cellular behavior. As shown by the comparative proteome analysis of $E$. coli cultures at either 0.1 or $0.2 \mathrm{~h}^{-1}$, proteome changes were significant not only in cellular activities known to be the hallmark of the stringent response, such as translation, transcription, and amino acid biosynthetic processes, but also in stress-related and transport activities. Proteome changes induced by the gene mutation at specific growth conditions highlighted cellular processes, such as transport and translation, suggesting that these processes are central during that steady-state conditions and are under the RelA-dependent stringent control. For example, it is likely that the downregulation of transporters in mutant cultures at a dilution rate of $0.1 \mathrm{~h}^{-1}$ is associated with the catabolite repression phenomena, which has been reported to be shut-off during the stringent response. Changes in protein expression levels provided evidences for the involvement of the RelA activity in a wide range of processes that resulted in structurally different regulatory modules across growth conditions. These modules represent the extent of differentially expressed protein that can be interrelated by sharing

\section{REFERENCES}

Barker, M. M., Gaal, T., Josaitis, C. A., and Gourse, R. L. (2001). Mechanism of regulation of transcription initiation by ppGpp. I. Effects of ppGpp on transcription initiation in vivo and in vitro. J. Mol. Biol. 305, 673-688. doi:10.1006/jmbi.2000. 4327

Brückner, R., and Titgemeyer, F. (2002). Carbon catabolite repression in bacteria: choice of the carbon source and autoregulatory limitation of sugar utilization. FEMS Microbiol. Lett. 209, 141-148. doi:10.1016/S0378-1097(02) 00559- 1

Carneiro, S., Villas-Bôas, S., Ferreira, E., and Rocha, I. (2012). Influence of the RelA activity on E. coli metabolism by metabolite profiling of glucose-limited chemostat cultures. Metabolites 2, 717-732. doi:10.3390/ metabo2040717

Carneiro, S., Villas-Bôas, S. G., Ferreira, E. C., and Rocha, I. (2011). Metabolic footprint analysis of recombinant Escherichia coli strains during fed-batch fermentations. Mol. Biosyst. 7, 899-910. doi:10.1039/c0mb00143k

Chang, D.-E., Smalley, D. J., and Conway, T. (2002). Gene expression profiling of Escherichia coli growth transitions: an expanded stringent response model. Mol. Microbiol. 45, 289-306. doi:10.1046/j.1365-2958.2002. 03001.x common regulators and become potential hubs in the stringent control.

In conclusion, the single relA gene mutation affects a large range of cellular processes even when cells are grown under steady-state conditions. The RelA-dependent response in E. coli cells is not only triggered by sudden environmental shifts, such as starvation conditions or diauxie transitions, but is also central to maintain many cellular activities in steady-state growth cultures. This confers to the RelA a role in processes to maintain tight control over all of the proteins needed for cell growth.

\section{AUTHOR CONTRIBUTIONS}

SC, EF, and SV-B designed the research. SC and IR performed the analyses. All authors interpreted the results and wrote the manuscript.

\section{ACKNOWLEDGMENTS}

The authors thank to Katie Smart for performing acetate analyses and the personnel of the Centre for Genomics and Proteomics of the University of Auckland for their excellent technical services and especially to Martin Middleditch for his diligent support in proteomic analysis.

\section{FUNDING}

This study was supported by the Portuguese Foundation for Science and Technology (FCT) under the scope of the strategic funding of UID/BIO/04469/2013 unit and COMPETE 2020 (POCI-01-0145-FEDER-006684).

\section{SUPPLEMENTARY MATERIAL}

The Supplementary Material for this article can be found online at http://journal.frontiersin.org/article/10.3389/fbioe. 2016.00078

Chong, P. K., Gan, C. S., Pham, T. K., and Wright, P. C. (2006). Isobaric tags for relative and absolute quantitation (iTRAQ) reproducibility: implication of multiple injections. J. Proteome Res. 5, 1232-1240. doi:10.1021/ pr060018u

Durfee, T., Hansen, A.-M., Zhi, H., Blattner, F. R., and Jin, D. J. (2008). Transcription profiling of the stringent response in Escherichia coli. J. Bacteriol. 190, 1084-1096. doi:10.1128/JB.01092-07

Ferullo, D. J., and Lovett, S. T. (2008). The stringent response and cell cycle arrest in Escherichia coli. PLoS Genet. 4:e1000300. doi:10.1371/journal.pgen. 1000300

Hauryliuk, V., Atkinson, G. C., Murakami, K. S., Tenson, T., and Gerdes, K. (2015). Recent functional insights into the role of (p)ppGpp in bacterial physiology. Nat. Rev. Microbiol. 13, 298-309. doi:10.1038/nrmicro3448

Herman, A., Wegrzyn, A., and Wegrzyn, G. (1994). Differential replication of plasmids during stringent and relaxed response of Escherichia coli. Plasmid 32, 89-94. doi:10.1006/plas.1994.1049

Jain, V., Kumar, M., and Chatterji, D. (2006). ppGpp: stringent response and survival. J. Microbiol. 44, 1-10. doi:10.1104/pp.106.077123.and

Kanjee, U., Ogata, K., and Houry, W. A. (2012). Direct binding targets of the stringent response alarmone (p)ppGpp. Mol. Microbiol. 85, 1029-1043. doi:10.1111/j.1365-2958.2012.08177.x 
Karp, N. A., Huber, W., Sadowski, P. G., Charles, P. D., Hester, S. V., and Lilley, K. S. (2010). Addressing accuracy and precision issues in iTRAQ quantitation. Mol. Cell. Proteomics 9, 1885-1897. doi:10.1074/mcp.M900628MCP200

Kristjansdottir, K., and Kron, S. J. (2010). Stable-isotope labeling for protein quantitation by mass spectrometry. Curr. Proteom. 7, 144-155. doi:10.2174/ 157016410791330552

Li, W., Bouveret, E., Zhang, Y., Liu, K., Wang, J. D., and Weisshaar, J. C. (2016). Effects of amino acid starvation on RelA diffusive behavior in live Escherichia coli. Mol. Microbiol. 99, 571-585. doi:10.1111/mmi.13252

Martin, D., Brun, C., Remy, E., Mouren, P., Thieffry, D., and Jacq, B. (2004), GOToolBox: functional analysis of gene datasets based on gene ontology. Genome Biol. 5, R101. doi:10.1186/gb-2004-5-12-r101

Miller, G. L. (1959). Use of dinitrosalicylic acid reagent for determination of reducing sugar. Anal. Chem. 31, 426-428. doi:10.1021/ac60147a030

Nanchen, A., Schicker, A., Revelles, O., and Sauer, U. (2008). Cyclic AMPdependent catabolite repression is the dominant control mechanism of metabolic fluxes under glucose limitation in Escherichia coli. J. Bacteriol. 190, 2323-2330. doi:10.1128/JB.01353-07

Paul, B. J., Ross, W., Gaal, T., and Gourse, R. L. (2004). rRNA transcription in Escherichia coli. Annu. Rev. Genet. 38, 749-770. doi:10.1146/annurev.genet.38. 072902.091347

Peterson, C. N., Mandel, M. J., and Silhavy, T. J. (2005). Escherichia coli starvation diets: essential nutrients weigh in distinctly. J. Bacteriol. 187, 7549-7553. doi:10.1128/JB.187.22.7549-7553.2005

Rocha, I., and Ferreira, E. (2002). On-line simultaneous monitoring of glucose and acetate with FIA during high cell density fermentation of recombinant $E$. coli. Anal. Chim. Acta 462, 293-304. doi:10.1016/S0003-2670(02)00347-1

Salgado, H., Martínez-Flores, I., López-Fuentes, A., García-Sotelo, J. S., PorrónSotelo, L., Solano, H., et al. (2012). Extracting regulatory networks of Escherichia coli from RegulonDB. Methods Mol. Biol. 804, 179-195. doi:10.1007/978-161779-361-5_10

Savitski, M. M., Fischer, F., Mathieson, T., Sweetman, G., Lang, M., and Bantscheff, M. (2010). Targeted data acquisition for improved reproducibility and robustness of proteomic mass spectrometry assays. J. Am. Soc. Mass Spectrom. 21, 1668-1679. doi:10.1016/j.jasms.2010.01.012

Schneider, D. A., Ross, W., and Gourse, R. L. (2003). Control of rRNA expression in Escherichia coli. Curr. Opin. Microbiol. 6, 151-156. doi:10.1016/S1369-5274(03) 00038-9
Schwacke, J. H., Hill, E. G., Krug, E. L., Comte-Walters, S., and Schey, K. L. (2009). iQuantitator: a tool for protein expression inference using iTRAQ. BMC Bioinformatics 10:342. doi:10.1186/1471-2105-10-342

Schweder, T., Hofmann, K., and Hecker, M. (1995). Escherichia coli K12 relA strains as safe hosts for expression of recombinant DNA. Appl. Microbiol. Biotechnol. 42, 718-723. doi:10.1007/BF00171951

Srivatsan, A., and Wang, J. D. (2008). Control of bacterial transcription, translation and replication by (p)ppGpp. Curr. Opin. Microbiol. 11, 100-105. doi:10.1016/j. mib.2008.02.001

Traxler, M. F., Chang, D.-E., and Conway, T. (2006). Guanosine 3,5'bispyrophosphate coordinates global gene expression during glucose-lactose diauxie in Escherichia coli. Proc. Natl. Acad. Sci. U.S.A. 103, 2374-2379. doi:10.1073/pnas.0510995103

Traxler, M. F., Summers, S. M., Nguyen, H.-T., Zacharia, V. M., Hightower, G. A., Smith, J. T., et al. (2008). The global, ppGpp-mediated stringent response to amino acid starvation in Escherichia coli. Mol. Microbiol. 68, 1128-1148. doi:10.1111/j.1365-2958.2008.06229.x

Wendrich, T. M., Blaha, G., Wilson, D. N., Marahiel, M. A., and Nierhaus, K. H. (2002). Dissection of the mechanism for the stringent factor RelA. Mol. Cell 10, 779-788. doi:10.1016/S1097-2765(02)00656-

Wiese, S., Reidegeld, K. A., Meyer, H. E., and Warscheid, B. (2007). Protein labeling by iTRAQ: a new tool for quantitative mass spectrometry in proteome research. Proteomics 7, 340-350. doi:10.1002/pmic.200600422

Wu, J., and Xie, J. (2009). Magic spot: (p) ppGpp. J. Cell. Physiol. 220, 297-302. doi:10.1002/jcp. 21797

Xiao, H., Kalman, M., Ikehara, K., Zemel, S., Glaser, G., and Cashel, M. (1991). Residual guanosine 3',5'-bispyrophosphate synthetic activity of relA null mutants can be eliminated by spoT null mutations. J. Biol. Chem. 266, 5980-5990.

Conflict of Interest Statement: The authors declare that the research was conducted in the absence of any commercial or financial relationships that could be construed as a potential conflict of interest.

Copyright $\odot 2016$ Carneiro, Villas-Bôas, Ferreira and Rocha. This is an open-access article distributed under the terms of the Creative Commons Attribution License (CC $B Y)$. The use, distribution or reproduction in other forums is permitted, provided the original author(s) or licensor are credited and that the original publication in this journal is cited, in accordance with accepted academic practice. No use, distribution or reproduction is permitted which does not comply with these terms. 